\title{
Effects of infliximab local injection on orthodontic tooth movement in diabetic rabbits: A histological study
}

Received: 19/4/2015

Accepted: 4/10/2015

\author{
Anees M. Mudhir * \\ Rafah Al-Maroof $* *$ \\ Fadhil Y. Jasim $* * *$
}

Abstract

Background and objective: Orthodontic tooth movement is mediated by tumor necrosis factor $-\alpha$ through activation of osteoclasts. Type 1 diabetes mellitus is characterized by a change in the bone remodeling and affects orthodontic treatment. This study aimed to investigate the effects of infliximab local injection on orthodontic movement in the diabetic rabbit.

Methods: Sixty six rabbits were divided equally into three groups; two groups were rendered diabetic using alloxan injection, while the third group was regarded as a normoglycemic group. Only one of the diabetic groups received insulin treatment. All rabbits were subjected to orthodontic tooth movement of upper central incisor for 18 days, half of each group received infliximab subperiosteal injection, while the remaining number received saline injection. After sacrifice, tissue samples were subjected to histological preparation and histomorphometric analysis.

Results: Insulin treated group revealed less prominent destruction of the periodontium when compared with the untreated group. The histological pictures of infliximab injected groups demonstrated less bone resorption in pressure side and more bone formation in tension side. There was a significantly smaller number of osteoclast with less compression in the width of the periodontal ligament in the tension side with infliximab injection.

Conclusion: Infliximab local injection hindered bone resorption in the diabetic state during orthodontic tooth movement.

Keywords: Infliximab; Orthodontic movement; Diabetes Mellitus.

\section{Introduction}

Diabetes mellitus has been associated with the occurrence of a series of complications like increased risk for osteoporosis, poor osseous healing, and impaired bone regeneration potential. ${ }^{1}$ The change in the metabolic state interferes with bone remodelling and results in a different rate of tooth movement. ${ }^{2}$ Braga et al. $^{3}$ reported greater tooth movement with retarded osseous regeneration, debilitation of the periodontal ligament, and microangiopathies in diabetic experimental mice when compared with the normal group. Diabetes affects the periodontium through cytokine dysregulation, where poor glycemic control is significantly correlated with the greater production of cytokines and higher cytokine levels in gingival fluid. ${ }^{4}$ Tumor necrosis factor-alpha (TNF- $\alpha$ ) is a potent immunomediator and proinflammatory cytokine that is rapidly up regulated after injury and secreted by macrophage, monocyte, and neutrophile. ${ }^{5}$ It exerted deleterious effects on glucose homeostasis and lipid metabolism and proposed to be causatively involved in the evolution of insulin resistance. ${ }^{6}$ TNF- $\alpha$ modifying processes is directly associated with tooth movement, and may induce mediators of the inflammatory reaction which will then impact osteoclast recruitment and function, suggesting a crucial role for TNF- $\alpha$ in orthodontic tooth movement. $^{7}$ Infliximab is a monoclonal antibody against TNF- $\alpha$ that binds to

* Department of orthodontic, College of Dentistry, Duhouk University, Duhouk, Iraq.

** Department of oral diagnosis, College of Dentistry, Hawler Medical University, Erbil, Iraq.

*** Department of orthodontic, College of Dentistry, Mosul University, Mosul, Iraq. 
soluble and mem-brane-bound TNF- $\alpha$ with a high compatibility and inhibits its effect by blocking TNF receptors interaction. The treatment with infliximab results in significant decrease in the inflammatory markers and inhibits bone destruction effectively. ${ }^{8}$ Andrade et al. ${ }^{9}$ showed that TNF- $\alpha$ antagonist significantly reduce the number of osteoclastic cells and controlling the production of chemokines in nonhuman primate model after the use of the orthodontic appliance in Wild-type mice (WT)and TNF-RI deficient mice. This study was conducted to investigate the role of local infliximab (TNF- $\alpha$ antagonist) injection on the periodontium of orthodontically moved teeth in experimentally induced diabetic rabbits.

\section{Methods}

A total of 66 male adult local breeded rabbits, weighting (1.5-2 $\mathrm{kg}$ ) were used in this study. After obtaining the approval from the ethical committee of Hawler Medical University, 44 rabbits received alloxan intravenous injection $(100 \mathrm{mg} / \mathrm{Kg})$ to induce diabetes mellitus. ${ }^{10}$ The injected rabbits were considered to have diabetes if fasting glucose level was $>300 \mathrm{mg} / \mathrm{dL}$. ${ }^{1}$ The blood glucose level was monitored every three days till the time of sacrifice using a glucose meter. Five days after confirming diabetes, 22 rabbits received subcutaneous insulin treatment $(0.5 \mathrm{IU} / \mathrm{Kg})$ every 48 hours in the morning till the animals were sacrificed. $^{10}$ Two months later, all 66 rabbits wear orthodontic appliance for 18

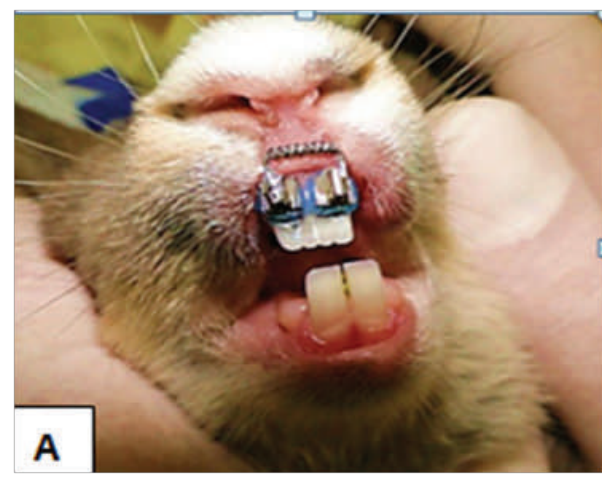

days. The appliance was consisting of two mini buccal tubes with $2.4 \mathrm{~mm}$ length and $2 \mathrm{~mm}$ diameter, slot $0.018^{*} 0.028$ inch. The tubes were bonded to the labial surface of rabbit upper central incisors almost in the cervical third of the clinical crown. L-shaped stainless steel wire of $0.017^{\star} 0.025$ inch with long arm $5 \mathrm{~mm}$ was inserted in the mini tube, while the short arm $3.5 \mathrm{~mm}$ faced the other short arm arch wire of the other mini buccal tube on the other upper central incisor. The force (approximately $35 \mathrm{gm}$ ) was applied by light strength nickel-titanium open coil spring fitted over the two horizontal wires sections with 0.079 inch diameter (Figure 1a). Half of each group (11rabbits) received a subperiosteal local injection of $0.1 \mathrm{ml}$ TNF- $\alpha$ antagonist on the labial side of orthodontically moved teeth. The infliximab was prepared by mixing the $50 \mathrm{mg}$ infliximab tablet with $10 \mathrm{ml}$ normal saline to end up with a concentration of $5 \mathrm{mg} / \mathrm{ml} .^{11}$ The remaining half (33 rabbits) from both normoglycemic and diabetic groups received $0.1 \mathrm{ml}$ of normal saline in the same manner using disposable insulin syringe (Figure 1b). The local injection was given at three different time intervals $\left(1^{\text {st }}, 7^{\text {th }}\right.$ and $13^{\text {th }}$ day) following the insertion of the appliance. After 18 days following appliance insertion, all rabbits were sacrificed humanely and the alveolar bone containing the moved teeth was subjected to H\&E (Haematoxylin \& Eosin) preparation and histomorphometric analysis using Motic digital microscope.

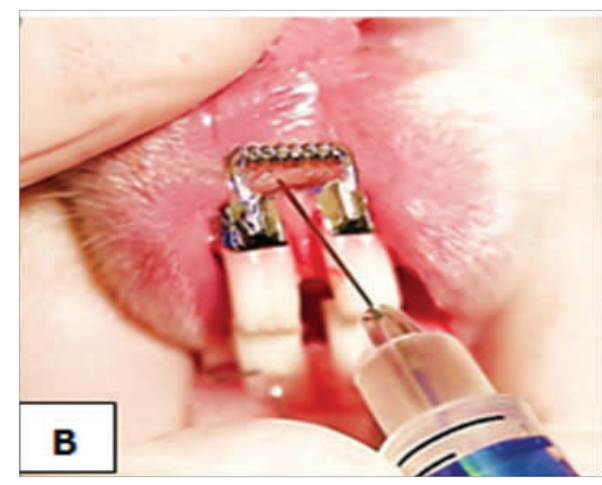

Figure 1: a- orthodontic appliance on upper central incisors before activation. b- Infliximab and normal saline local injection site after activation. 
http://dx.doi.org/10.15218/zjms.2016.0029

The width of the PDL on the tension side was measured $(\mathrm{x} 100)$ in three different areas (cervical, middle, and apical area) and the mean was recorded and subjected to the statistical analysis. On the pressure side, counting of osteoclasts on the periphery of the alveolar bone was done under high power (x400) that meet the criteria of multinucleated giant cells rested in Howship's lacunae. Means and standard deviations, one way analysis of variance test ANOVA and Pairwise multiple comparison procedures (HolmSidak method) were used to compare all groups using the statistical package for the social sciences (version 17). $P$ value $\leq 0.05$ was considered statistically significant.

\section{Results}

1-The normoglycemic group injected with saline (NG): On examining the tension side of the moving tooth, there was an obvious increase in the width of the PDL as a result of orthodontic movement with the formation of many newly formed bony projections that were extended in the same direction of the tooth movement. The staining intensity of these newly formed bony projections obviously differs from the old bone with the presence of well demarcated resting line. The bony projections incorporated many large nuclei osteocytes within their lacunae and their peripheries were lined by a large population of osteoblasts. The PDL demonstrated high cellularity with the disorientation of its principle fibers especially next to the bone surface (Figure $2 \mathrm{~A}$ and $\mathrm{B}$ ). On the pressure side, the alveolar bone lost the smooth bundle bone surface, instead, alveolar bone demonstrated irregular outline as a result of the presence of many depressions that were occupied by multinucleated giant osteoclasts especially on the internal side. The PDL principle fibers appeared to arrange parallel to the tooth long axis with inactive fibroblasts run in the same direction and compressed nuclei with scanty cytoplasm and most of the interstitial spaces were in close contact with the periphery of the bone (Figure $2 \mathrm{C}$ and D).

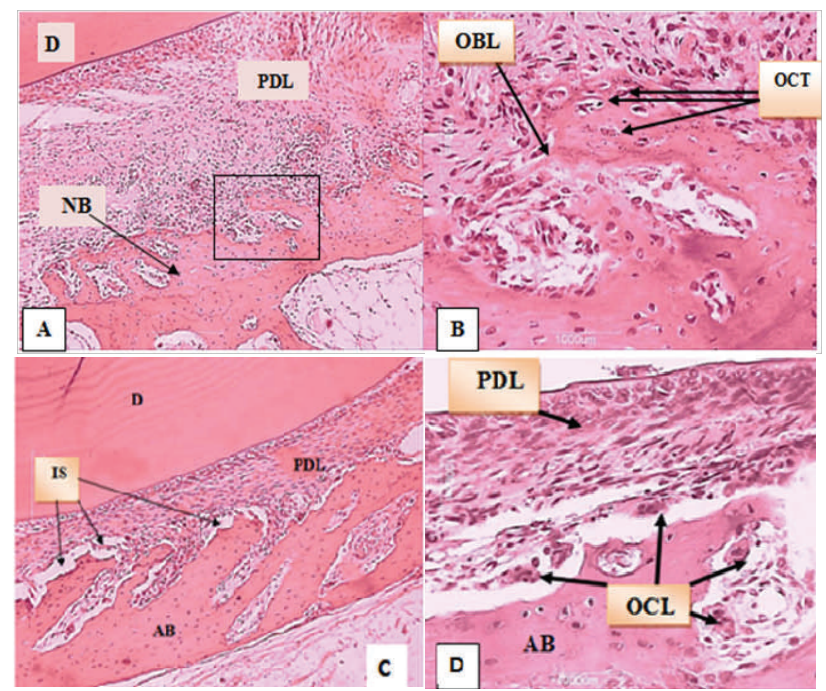

Figure 2: Microphotograph of the normoglycemic group injected with saline. A: Tension side demonstrates formation of new bony projections (NB), PDL highly cellular. B: Magnification of the square area bony projections lined by osteoblasts and incorporated many osteocytes. C: Pressure side shows compressed PDL with fibers run parallel to the tooth surface and irregular alveolar bone periphery with interstitial spaces (IS) close to it. D: osteoclasts in the internal \& external bone surface of the pressure side. (H\&E. A: $x 100$, B: $x 400, C: x 100, D: x 400) . A B$ : alveolar bone, PDL: periodontal ligament, D:dentin, OBL: osteoblast; OCT: osteocyte. 
http://dx.doi.org/10.15218/zjms.2016.0029

2- The normoglycemic group injected with infliximab(NGF): The tension side demonstrated wide PDL filled with many blood vessels and thin newly formed bone spicules extended in the same direction of tooth movement and incorporated many osteocytes within their lacunae that sometimes contained more than one nucleus. The bony spicules were lined by a large number of osteoblasts and the attachment of the PDL principle fibers to the bony spicules was very clear (Figure $3 \mathrm{~A}$ and $\mathrm{B}$ ). On the pressure side, the principle fibers were parallel to the long axis of the tooth. The amount of bone resorption was less than in the normoglycemic group with saline injection as indicated by the smooth periphery of the bone, smaller size and number of the osteoclasts (Figure $3 \mathrm{C}$ and D).
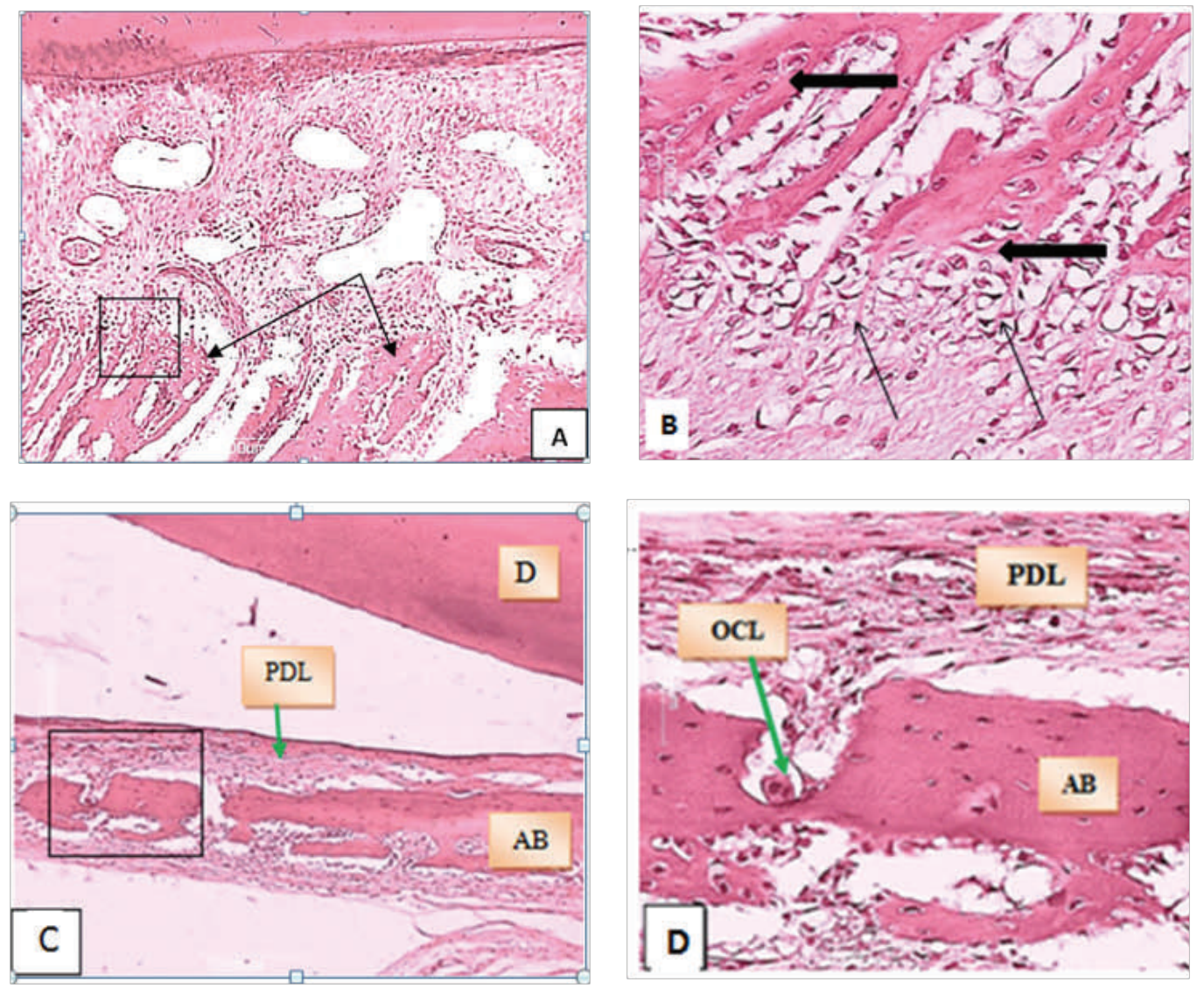

Figure 3: Microphotograph of the normoglycemic group injected with infliximab. A:In tension side, wide PDL filled with blood vessels and extended bony projections (arrows). B: Magnification of square area shows Sharpey's fibers extended between strands of osteoblasts (thin arrows) \& interrupted osteocytes (thick arrows). C: Compressed PDL with principle fibers parallel to the tooth long axis D: Magnification of square area represent site of bone resorption with a depression occupied by osteoclast (OCL) (H\&E A: x100, B: x400, C: x40, D: x400). D: dentin, AB: alveolar bone, PDL: periodontal ligament. 
http://dx.doi.org/10.15218/zjms.2016.0029

3. The uncontrolled diabetic group injected with saline (UCD): The periodontium of uncontrolled diabetic demonstrated enormous histological changes. The PDL on the tension side showed massive widening with loosening of the principle fibers that accompanied by many empty spaces between the collagen fibers unlike the previous group. Despite that, the formation of new bony trabeculae was still detected. The most prominent change observed in this group was the blood vessels, were many locations demonstrated destructed walls of blood vessels with extravasations of R.B.C (Figure $4 \mathrm{~A}$ and $\mathrm{B}$ ). On the pressure side, the destruction of the blood vessels wall was also noticed on this side with infiltration of many inflammatory cells. Bone resorption was recorded on the periphery of the bone with irregular surfaces and presence of Howship's lacunae that were more prominent on the internal surfaces of the marrow cavity (Figure $4 \mathrm{C}$ and D).
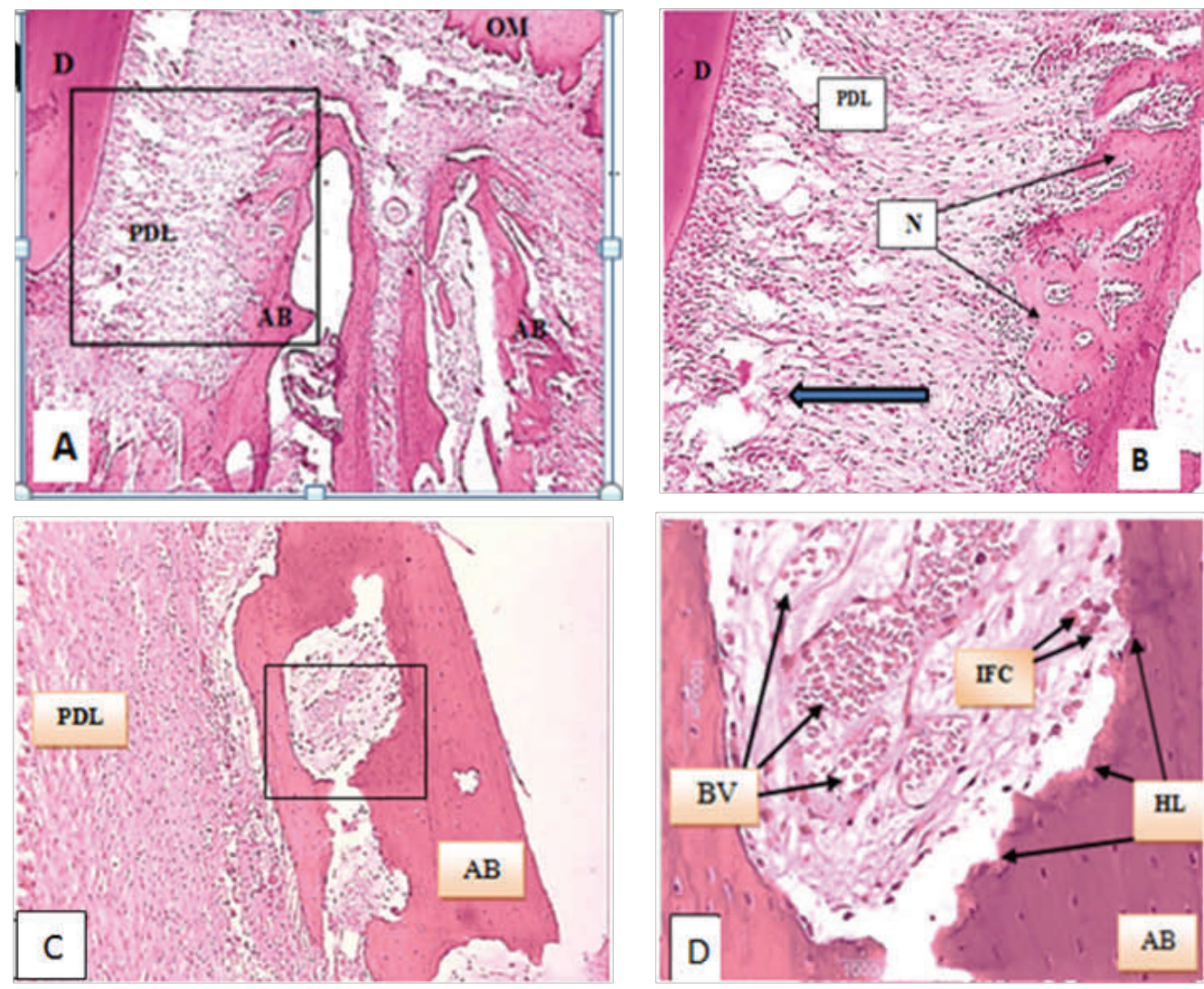

Figure 4: Microphotograph of uncontrolled diabetic rabbit injected with normal saline. A:Tension side demonstrated loosening of the principle fibers accompanied by extended new bone trabeculae. B: Magnification of square area shows the newly formed bone $(\mathrm{N})$ fainter than old bone $(\mathrm{OB})$ and many destructed blood vessels (Thick arrow). C: Compressed PDL with extravasation of R.B.C. D: Magnification of square area, the marrow cavity filled with blood vessels and infiltrated by many inflammatory cells (IFC).. (H\&E. A:x40, B:x100, C:x40, D:x400).AB: alveolar bone; BV: blood vessels, HL: Howships lacunae, D: dentin, OM: oral mucosa. 
4. The uncontrolled diabetic group injected with infliximab (UCDF): In tension side, the principle fibers of PDL preserve most of its normal appearance with the presence of thick wavy bundles that most of them directed obliquely. The principle fibers inserted in cementum in a perpendicular direction with the presence of many active fibroblasts between them. The PDL appeared to be less destructed than the previous saline group and the newly formed bony projection had a smoother surface that lined by many osteoblasts. The new bone was fainter and easily noticed even under low power examination.
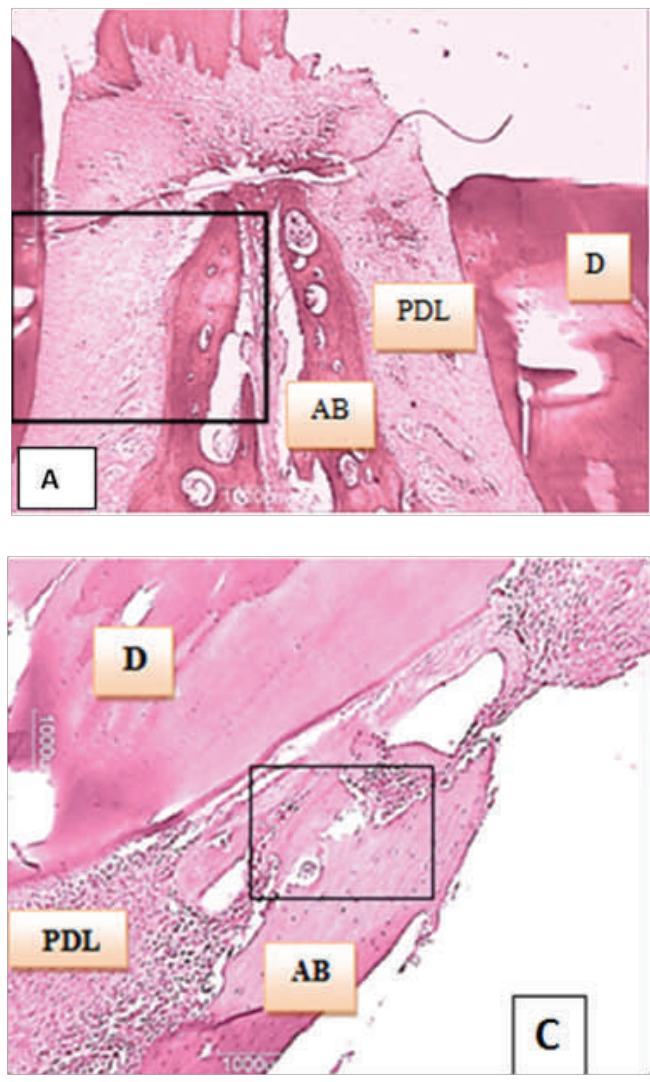

The degree of inflammation appeared to be less prominent than in the uncontrolled diabetic group with saline injection although many congested blood vessels were present within the interstitial spaces. (Figure $5 \mathrm{~A}$ and $\mathrm{B}$ ). As in other groups, the PDL on the pressure side was compressed with the principle fiber ran parallel to the tooth long axis with smoother surface of the alveolar bone when compared with group injected with saline. The internal resorption was noticed with presences of many Howship's lacunae that housed multinucleated giant cells (Figure $5 \mathrm{C}$ and D).
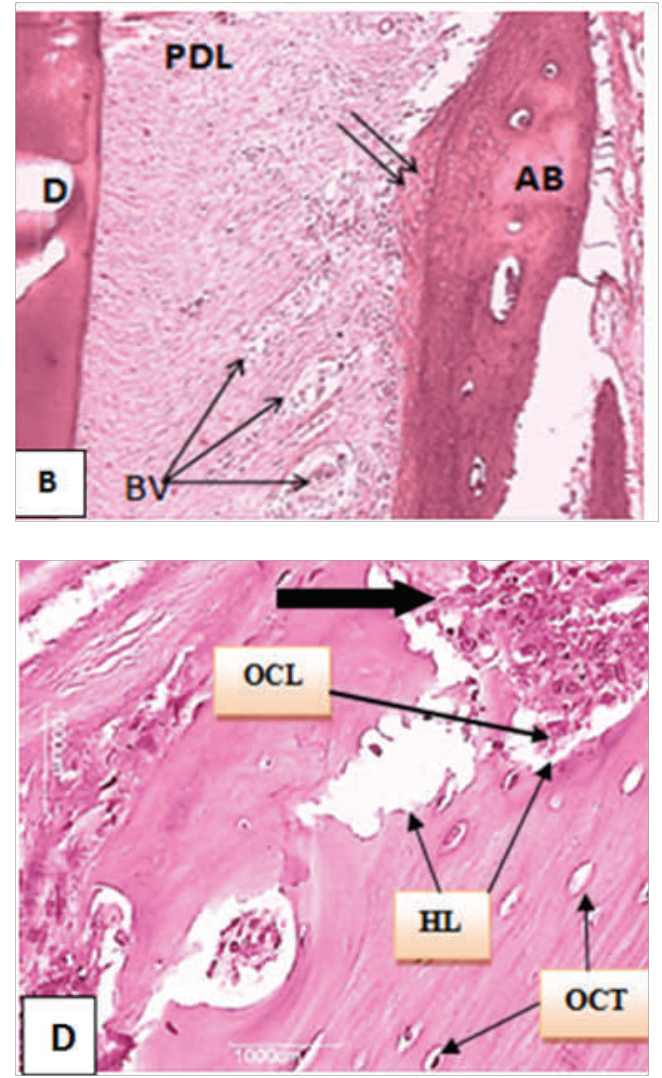

Figure 5: Microphotograph of uncontrolled diabetic rabbit injected with infliximab. A: Tension side shows smooth alveolar bone with well condensed PDL. B: Magnification of square area, the newly formed bone( double arrow) is fainter and PDL contain many congested blood vessels. C: Pressure side shows irregular periphery of the bone with Howships lacunae containing osteoclasts. D: Magnification of the square area shows many osteoclasts and marrow cavities filled with inflammatory cells (Thick arrow).(H\&E: A:x40, B: x100, C:x40, D:400). D:dentin, AB: alveolar bone, BV: blood vessel, OCL:osteoclast, OCT: osteocyte, HL: Howship's lacunae. 
5- The controlled diabetic group injected with saline (CD): On the tension side, the principle fibers were extended between the bone and cementum with obvious preservation of their normal structures especially their attachment to the bone surface. The Sharpey's fibers were thick and easily detected even under low power. Newly formed bone spicules were longer \& wider than uncontrolled group. The PDL greatly differ from the uncontrolled diabetic group. It was more fibrous and the collagen fibers were more condensed with a chance to localized many congested blood vessels (Figure $6 \mathrm{~A}$ and $\mathrm{B}$ ). The PDL in the pressure side was compressed and contained many blood vessels that showed some signs of destruction with extravasation of R.B.C. The periphery of the bone demonstrated irregular outlines as a result of the presence of much Howship lacunae that contain multinucleated giant cells (Figure 6 C and D).
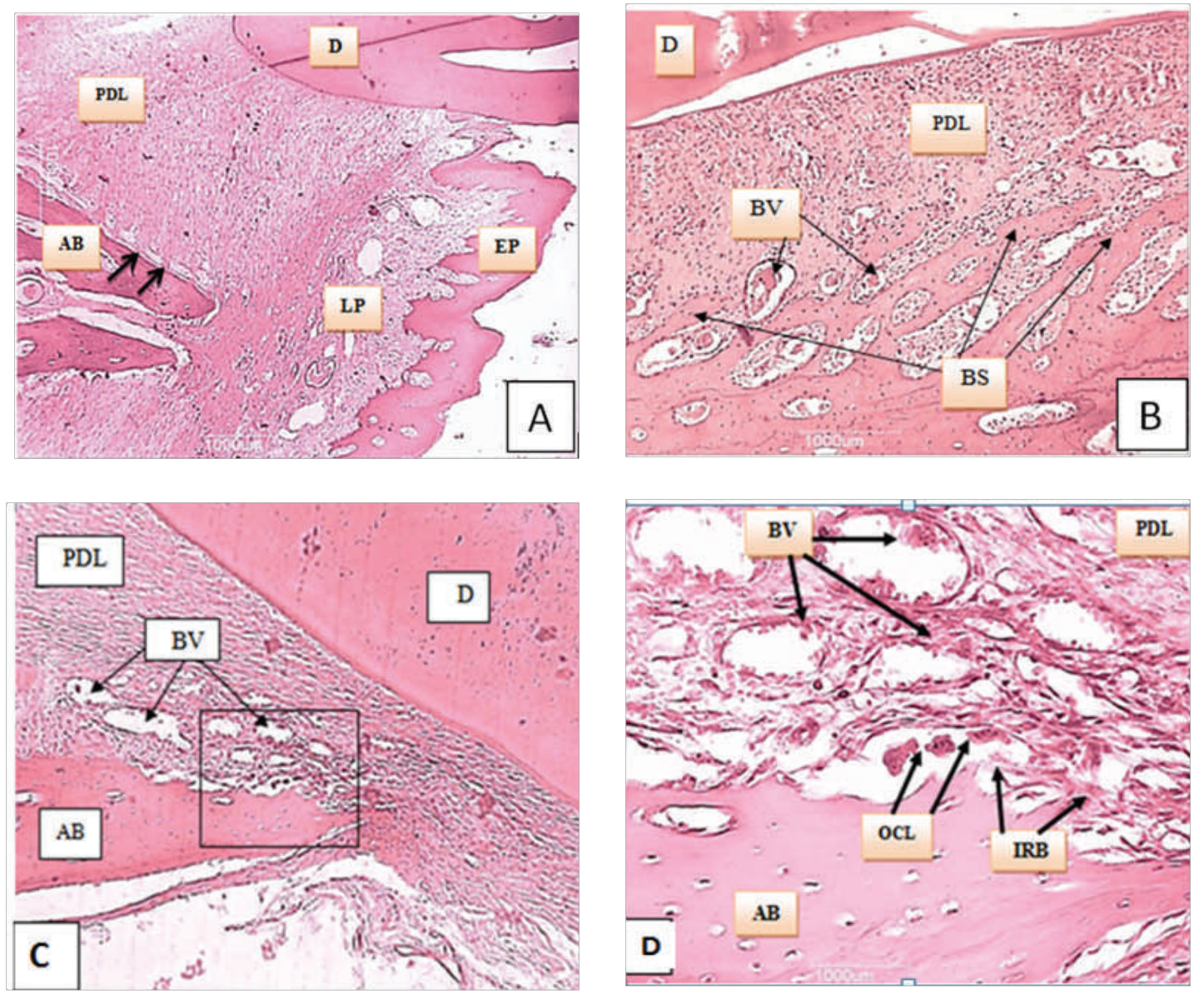

Figure 6: Microphotograph of controlled diabetic rabbit injected with normal saline. A: Tension side shows well preserved PDL features with thick Sharpey's fibers on the bone side. B: Presence of newly formed bone spicules lined by osteoblast and filled with osteocytes. PDL filled with congested blood vessels. C: Pressure side bone periphery demonstrated irregular outlines with the presence of Howship's lacunae. D: Magnification of the square area, many congested blood vessels \& osteoclasts. (H\&E. A:x40, B:x100, C:x100, D:x400). D: dentin; AB: alveolar bone, EP: epithelium, LP: lamina properia, BV: blood vessels, BS: bone spicules, IRB: irregular outline bone; OCL: osteoclast. 
6. The controlled diabetic group injected with infliximab (CDF): The treatment of diabetic rabbits with insulin hindered the histological changes that were more prominent in the saline untreated group. The inflammatory process was less recognized and the arrangement of the collagen fiber was preserved and well organized with intact Sharpey's fiber. Despite that, the interstitial spaces still filled with large number of congested blood vessels. The bone of the alveolar process demonstrated the formation of a new layer which was well distinguished from old bone and lined by well-arranged osteoblasts (Figure $7 \mathrm{~A}$ and $\mathrm{B}$ ). On the pressure side, the PDL fibers arranged parallel to the tooth long axis with compression of the interstitial spaces and compression of the fibroblasts especially in the middle region. The periphery of the bone showed signs of resorption with the presence of small osteoclasts in shallow Howship's lacunae. The bone resorption appeared to be less prominent than other groups even the normoglycemic group that received a saline injection (Figure $7 \mathrm{C}$ and $\mathrm{D}$ ).
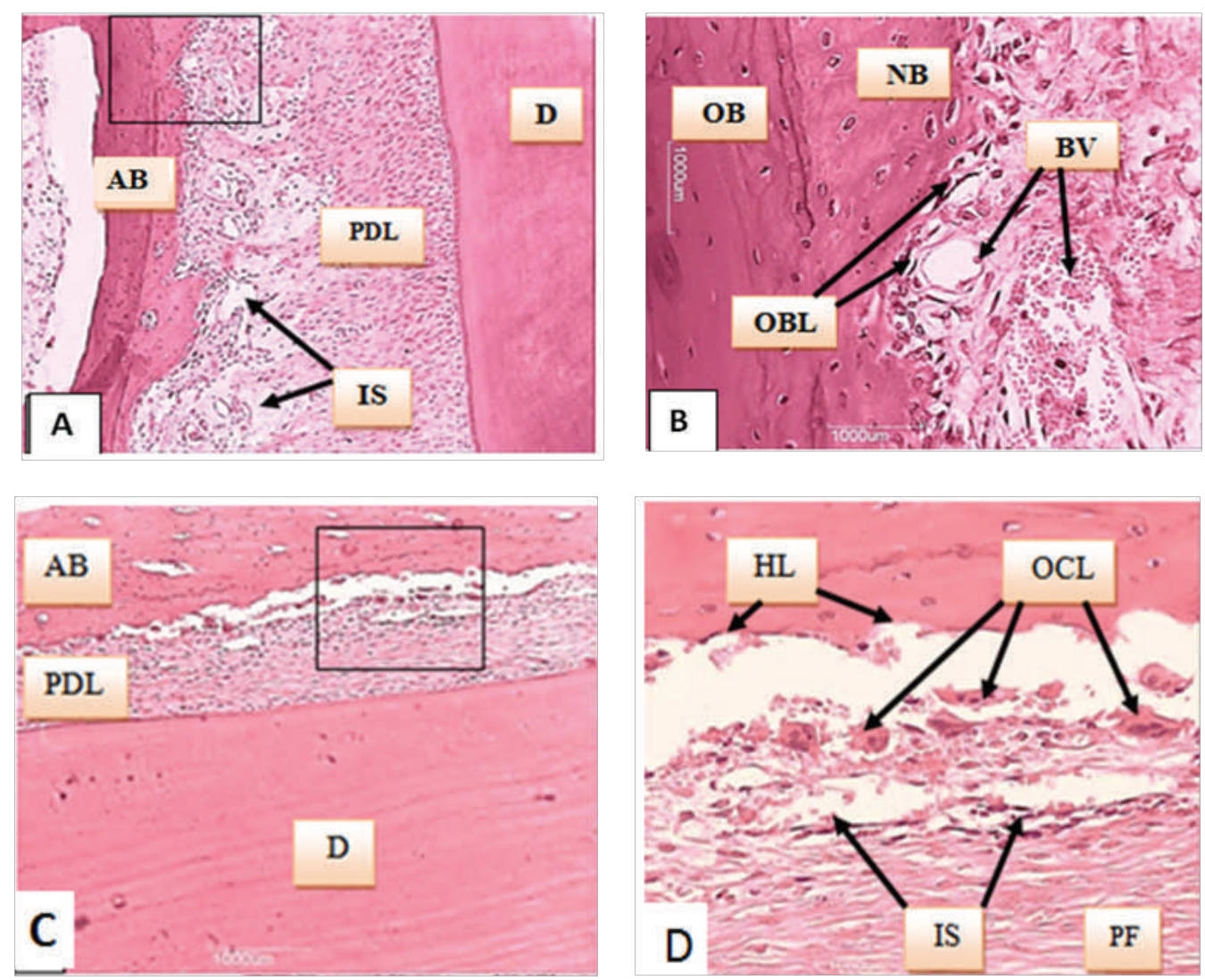

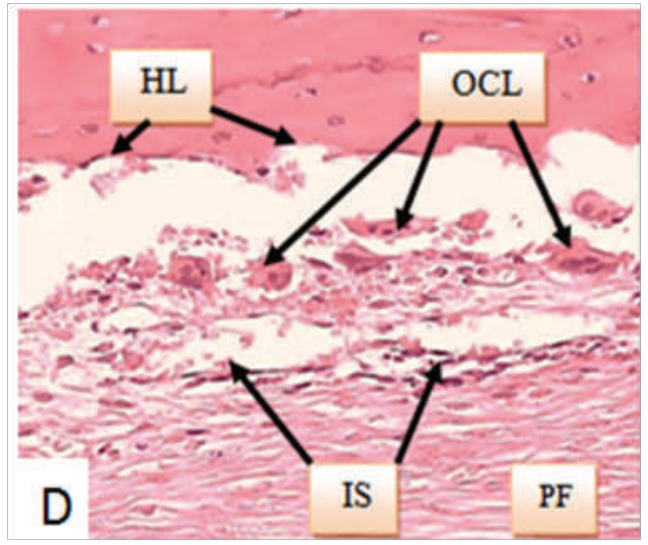

Figure 7: Microphotograph of controlled diabetic rabbit injected with infliximab. A: Tension side shows well-organized collagen fiber with intact Sharpey's. Bony projections lined by osteoblasts. B: Magnification of square area demonstrated new bone filled with osteocytes \& interstitial spaces filled with congested blood vessels. C: Irregular bone's periphery. D: Shallow Howship's lacunae occupied by osteoclasts (H\&E. A: x100, B: x400, C:x40, D:x400). AB:alveolar bone, D:dentin, IS: interstitial space, OB: old bone, NB: new bone, BV: blood vessels OCL:osteoclast, PF:principle fiber, HL: Howship's lacunae. 


\section{Histomorphometric analysis}

1-Periodental ligament (PDL) width on the tension side: Baseline data were collected from 4 normoglycemic rabbits that didn't undergo any orthodontic treatment. The means and the standard deviations of PDL width for all studied groups are shown in Table 1. The width of PDL was changed in all groups when compared with normal baseline data that was $(49.82 \pm 15.83 \mu \mathrm{m})$. The highest increase in the width of PDL was observed in the uncontrolled diabetic group (UCD) which was $(231.08 \pm 42.57 \mu \mathrm{m})$, while the least change was seen in the normoglycemic group that received infliximab injection (NGF), where PDL width was $(84.28 \pm 14.45 \mu \mathrm{m})$. Generally, infliximab injection caused an obvious reduction in the width of PDL even in uncontrolled diabetic group. On performing ANOVA test, the subperiosteal infliximab injection caused significant differences only in the diabetic group whether uncontrolled or controlled. The diabetic state affected the PDL width with high significant level in both, uncontrolled or controlled as shown in Table 2. 2-Osteoclast number: The highest number of osteoclast was observed in uncontrolled diabetic group injected with normal saline (UCD) (24.36 \pm 5.25$)$, while the least number was observed in the controlled diabetic group that received infliximab injection(CDF), which was (7.91 \pm 3.91$)$. All groups that were injected with infliximab demonstrated a smaller number of osteoclasts in comparing with saline injection groups as shown in Table 1.

Table 1: Means and standard deviations of PDL width in tension side in all study groups in $\mu \mathrm{m}$., and of osteoclast number in pressure side in all study groups.

\begin{tabular}{|c|c|c|c|}
\hline Groups & PDL width Mean \pm std $(\mu \mathrm{m})$ & Osteoclast Mean \pm SD & $\begin{array}{l}\text { P value } \\
\text { (ANOVA) }\end{array}$ \\
\hline Normal & $49.82 \pm 15.85$ & $8.90 \pm 2.34$ & \multirow{7}{*}{$<0.001$} \\
\hline NG & $114.14 \pm 26.89$ & $19.90 \pm 4.20$ & \\
\hline NGF & $84.27 \pm 14.45$ & $9.09 \pm 2.87$ & \\
\hline$C D$ & $200.23 \pm 30.06$ & $18.00 \pm 7.87$ & \\
\hline CDF & $137.68 \pm 65.80$ & $7.90 \pm 3.91$ & \\
\hline UCD & $231.07 \pm 42.57$ & $24.36 \pm 5.25$ & \\
\hline UCDF & $176.09 \pm 34.49$ & $13.81 \pm 4.44$ & \\
\hline
\end{tabular}

Table 2: One-way analysis of variance test for means and standard deviations of PDL width in the tension side in all study groups.

\begin{tabular}{|c|c|c|c|c|c|c|}
\hline Groups & NG & NGF & UCD & UCDF & $C D$ & CDF \\
\hline NG & & 29.86 & $116.93^{* *}$ & $61.95^{*}$ & $86.08^{* *}$ & 23.54 \\
\hline NGF & & & $146.80^{* *}$ & $91.82^{* *}$ & $115.95^{* *}$ & $53.41^{*}$ \\
\hline UCD & & & & $54.98^{*}$ & 30.84 & $93.39^{* *}$ \\
\hline UCDF & & & & & 24.13 & 38.41 \\
\hline$C D$ & & & & & & $62.54^{*}$ \\
\hline
\end{tabular}

$\mathrm{F}=34.218, P<0.001$

Holm-Sidak Pairwise multiple comparison procedures ( ${ }^{*}$ significant $P<0.05$, ** highly significant $P<0.001$ ). 
On performing ANOVA test, the infliximab injected grouped (normoglycemic and diabetic) showed a smaller number of osteoclasts with a high level of significancy when compared with corresponded groups injected with saline. On the other hand insulin treatment significantly affected osteoclasts number when compared with uncontrolled groups with or without infliximab local injection as shown in Table 3.

\section{Discussion}

Orthodontic tooth movement takes place through remodeling of alveolar bone as a result of the force that is exerted on the periodontium. ${ }^{3}$ This study was conducted to assess the effect of local administration of infliximab on tooth movement in normoglycemic and diabetic model in an attempt to interfere with TNF- $\alpha$ which is usually elevated during orthodontic treatment, ${ }^{9}$ and during diabetes mellitus. ${ }^{12}$ The orthodontic movement of rabbit's central incisor with infliximab injection caused many histological changes that were confirmed by histomorphometric results. The infliximab injection decreased the rate of tooth movement with a significantly smaller width of PDL in tension side and a smaller number of osteoclasts in pressure side. The infliximab effect was more pronounced in the diabetic group than in a normoglycemic group. Previous studies done by Blesta et al. $^{13}$ and SeonBaik et al. ${ }^{14}$ showed that orthodontic loading prompt the elevation of TNF- $\alpha$ and IL-1 that occurred within the first day following mechanical force application in both compression and tension areas. TNF- $\alpha$ is a typical mediator of inflammatory response that has been shown to be involved in the process of bone resorption. ${ }^{15}$ The results of this study may be due to the inhibition of TNF- $\alpha$ leading to increase in new bone matrix formation, since the presence of TNF- $\alpha$ antagonist cause significant reduction in apoptosis of bone-lining cells in diabetes, and, consequently, a less number of osteoclasts and less bone resorption. ${ }^{16}$ The arrangement of the collagen fibers was differed between the controlled and uncontrolled diabetic groups with more uniformed well-packed fibers with insulin treatment, this come in accordance with study done by Tawfiq $^{17}$ that showed the amount of collagen fibers in the wound done in the rat's tongue was lowered dramatically in diabetic rats than in the normal group. The same result documented in the study done by Xin et al., ${ }^{18}$ which used orthodontic therapy in diabetes induced rats for 14 days. Their study demonstrated significant lowering in the expression of collagen fibers in PDL of

Table 3: One-way analysis of variance test for means and standard deviations of osteoclasts number in the pressure side of all study groups.

\begin{tabular}{|c|c|c|c|c|c|c|}
\hline Groups & NG & NGF & UCD & UCDF & CD & CDF \\
\hline NG & & $10.81^{* *}$ & 4.45 & \multirow{2}{*}{$\begin{array}{l}6.09^{*} \\
4.72\end{array}$} & 1.90 & $12.00^{* *}$ \\
\hline NGF & & & $15.27^{* *}$ & & $8.90^{* *}$ & 1.18 \\
\hline UCD & & & & \multirow[t]{3}{*}{$10.54^{* *}$} & $6.36^{*}$ & $16.45^{\star *}$ \\
\hline UCDF & & & & & 4.18 & $5.90^{*}$ \\
\hline$C D$ & & & & & & $10.09^{* *}$ \\
\hline
\end{tabular}


adiabetic rats than in normoglycemic rats. The changes in collagen fibers may be due to hyperglycemia, a characteristic feature of diabetes, that inhibits the proliferation and differentiation of fibroblasts and contribute to the delayed periodontal regeneration and healing seen in patients with diabetes mellitus. ${ }^{19}$ Rapid bone resorption and marked PDL destruction are features of orthodontic movement in animal models of diabetes in a study done by $\mathrm{Li}$ et al., ${ }^{2}$ with significantly more osteoclasts in the PDL of diabetic induced rats compared with normoglycemic rats after orthodontic treatment. Graber et al. ${ }^{20}$ stated that TNF- $\alpha$ plays a prominent role in the mechanism controlling the appearance of osteoclasts at compression sites. An increase in the expression of TNF- $\alpha$ was observed in periodontal tissues surrounding teeth in diabetic mice more than in normoglycemic mice at 12 and 72 hours of orthodontic appliance mechanical loading which exhibited greater orthodontic tooth movement and higher number of osteoclasts during histomorphometric analysis. ${ }^{3}$ Tumor necrosis factor-alpha (TNF-a) is members of the formerly known osteoclast-activating factor that have been implicated in the bone remodeling process. $^{13}$

\section{Conclusions}

Infliximab injection around the orthodontically moved significantly decreased the rate of movement in normoglycemic and diabetic rabbits and decreased the destructive effect of diabetic disease with preservation of the periodontal tissue especially in the uncontrolled diabetic group. Local injection of infliximab could be a promising method for decreasing bone resorption and periodontal inflammation in diabetic rabbits undergoing orthodontic treatment.

\section{Conflicts of interest}

The authors report no conflicts of interest.

\section{References}

1. Retzepi M, Lewis MP, Donos N. Effect of diabetes and metabolic control on de novo bone formation following guided bone regeneration. Clin Oral Implants Res 2010; 21:71-9.

2. Li $\mathrm{X}$, Zhang $\mathrm{L}$, Wang $\mathrm{N}$, Feng $\mathrm{X}, \mathrm{Bi}$ L. Periodontal ligament remodeling and alveolar bone resorptionduring orthodontic tooth movement in rats with diabetes. Diabetes Technol Ther 2010; 12:65-73.

3. Braga SM, Taddei SR, Andrade IJ, QueirozJunior CM, Garlet GP, Repeke CE, et al. Effect of diabetes on orthodontic tooth movement in a mouse model. Eur J Oral Sci 2011; 119:7-14.

4. Engebretson SP, Hey-Hadavi J, Ehrhardt FJ, Hsu D, Celenti RS, Grbic JT, et al. Gingival crevicular fluid levels of interleukin-1beta and glycemic control in patients with chronic periodontitis and type 2 diabetes. J Periodontol 2004; 75:1203-8.

5. VanDoornum S, McColl G, Wicks IP. Tumour necrosis factor antagonists improve disease activity but not arterial stiffness in rheumatoid arthritis. Rheumatology (Oxford) 2005; 44: 1428-32.

6. Gonzalez-Gay MA, De Matias JM, Gonzalez-Juanatey C, Garcia-Porrua C, Sanchez -Andrade A, Martin J, et al. Anti-tumor necrosis factor-alpha blockade improves insulin resistance in patients with rheumatoid arthritis. Clin Exp Rheumatol 2006; 24:83-6.

7. Yoshimatsu M, Shibata $Y$, Kitaura H, Chang $X$, Moriishi T, Hashimoto $F$, et al. Experimental model of tooth movement by orthodontic force in mice and its application to tumor necrosis factor receptor- deficient mice. J Bone Min Metab2006; 24:20-7.

8. Korczowska I, Lacki JK, Hrycaj P. Influence of infliximab on cytokines network and markers of bone remodeling in rheumatoid arthritis patients. Yonsei Med J 2013; 54:183-8.

9. Andrade I, Silva TA, Silva GA, Teixeira AL, Teixeira MM. The role of tumor necrosis factor receptor type 1 in orthodontic tooth movement. J Dent Res 2007; 86:1089-94.

10. Ibrahim RS. Effect of low energy laser irradiation on bone healing around intra osseous titanium implant in experimentally diabetic rabbits. PhD thesis in Oral histology and biology. University of Baghdad. College of Dentistry; 2003.

11. Seneschal J, Lepreux S, Bouyssou-Gauthier ML, Heliot-Hosten I, Economu A, Dehais J, et al. Psoriasiform drug eruptions under anti-TNF treatment of arthritis are not true psoriasis. Acta Derm Venereol 2007; 87:77-80.

12. Southerland JH, Taylor GW, Offenbacher S. Diabetes and periodontal infection: making the Connection. Clin Diabetes 2005; 23:171-8.

13. Bletsa A, Berggreen E, Brudvik P. Interleukin-1a and tumor necrosis factor- $\alpha$ expression during the early phases of orthodontic tooth movement in rats. Eur J Oral Sci 2006; 114:423-9. 
14. SeonBaik H, Kwan-Kim CH, Lim W, Chun Y. Interleukin-1 $\alpha$ and tumor necrosis factor- $\alpha$ expression on the compressed side of gingiva during orthodontic tooth movement. OJST 2012; 2:182-7.

15. Kaya FA, Hamamci N, Basaran G, Dogru M, Yildirim TT. TNF- $\alpha, \mathrm{IL}-1 \beta$ and IL-8 levels in tooth early leveling movement orthodontic tratment. J Int Dent Med Res 2010; 3:116-21.

16. Liu R, Bal HS, Desta T, Behl Y, Graves DT. Tumor necrosis factor- $\alpha$ mediates diabetesenhanced apoptosis of matrix-producing cells and impairs diabetic healing. Am J Pathol 2006; 168:757-64.

17. Tawfiq FF. The effect of tumor necrosis factor- $\alpha$ inhibitor on the wound healing of oral mucosa in induced diabetic rats. M. Sc. thesis in Oral histology. Hawler Medical University; 2011.

18. Xie R, Kuijpers-Jagtman AM, Maltha JC. Osteoclast differentiation and recruitment during early stages of experimental tooth movement in rats. Eur J Oral Sci 2009; 117:43-50.

19. Kim HS, Park JW, Yeo SI, Choi BJ, Suh JY. Effect of high glucose on cellular activity of periodontal ligament cells invitro. Diabetes Res Clin Pract 2006; 74:41-7.

20. Graber LW, Vanarsdall Jr, R, Vig KW. Orthodontics current principles and techniques. $5^{\text {th }}$ ed. Philadelphia: Elsevier Mosby; 2012. 